\title{
The effect of electrode material and semicon bonding on space charge dynamics of XLPE
}

\author{
M. Hao, A. Fazal, A. S. Vaughan, G. Chen \\ Tony Davis High Voltage Laboratory \\ University of Southampton \\ Southampton, SO17 1BJ, UK \\ M.Hao@soton.ac.uk
}

\author{
J. Cao, H. Wang \\ Smart Grid Research Institute \\ Beijing, China
}

\begin{abstract}
This study sets out to explore the space charge characteristics of high voltage direct current (HVDC) cable grade crosslinked polyethylene (XLPE) using different electrode materials and configurations, ranging from sputtered gold to thermally bonded semi-conducting materials. The existence of both homo and hetero charges in HVDC cables is well known; however, the role of semi-conducting materials and the formation of space charge is critical and required more attention. Space charge accumulation at high electric stress was monitored using the pulsed electroacoustic (PEA) technique. Experimental results have shown that charge injection processes take place in all cases and the amount of charge and polarity of the dominated injected charge has significant dependence on the electrode material under the same applied electric field.
\end{abstract}

Keywords - Space charge; PEA; XLPE; HVDC cable; semiconducting material; charge injection

\section{INTRODUCTION}

Interest in high voltage direct current (HVDC) transmission has led many manufactures to develop in XLPE DC power cables. XLPE insulation has excellent dielectric strength and electrical resistivity as well as excellent physical properties including resistance to cracking and moisture penetration. This has resulted in XLPE becoming the most common polymeric insulation used in power cable transmission up to $500 \mathrm{kV}[1]$. However, there are drawbacks associated with XLPE, such as low charge carrier mobility and trapping rates (under certain conditions) giving a rise to space charge in the insulating material $[2,3]$. This can result in increasing localized electric stress and premature failure of the cable insulation [4-6]. It has been reported in the literature that space charge can be formed in polymeric insulation under DC conditions. When power cables are loaded under high DC voltage, an accumulation of space charge develops within the insulation material, which poses a threat to the reliability of the cable and is one of the causes of unexpected breakdown $[3,7]$. Therefore, the future development of HVDC polyethylene insulated cables requires a fundamental understanding of potential failure mechanisms.

In general, under laboratory conditions, electrodes such as gold or aluminum are sputter coated onto the sample so that an external voltage can be applied. However, the results derived from laboratory measurements may be vary from those in practice. Therefore, it is important to examine the influence of semicon on the charge formation. For space charge characteristics, the PEA method is the most widely used for HVDC cables. This technique utilizes the interaction between high voltage pulses and charge layers accumulated in the insulating material to produce acoustic pressure waves, which transverse across the material [8].

This paper reports on an investigation into the space charge formation of XLPE as insulation with different electrode materials. In particular, the influences of space charge formation and distribution in the system. By focusing on the PEA technique, in this work, an attempt is made to explore the space charge characteristics, using a spectrum of different electrode materials and configurations, ranging from the conventional setup to sputtered gold and thermally bonded semiconducting materials. Two sets of electrodes are considered (i) polymer semiconductive material (semicon, $\mathrm{Sc}$ ) and (ii) metallic, this includes aluminum (Al) and gold (Au). Al has been widely used in the laboratory based work and $\mathrm{Sc}$ is employed in power cables and for this reason both types of electrodes have been investigated.

\section{EXPERIMENTAL}

\section{A. Materials Considered}

Bulk samples were produced from an HV cable grade low density polyethylene (LDPE) material and the carbon loaded semicon was produced using the same grade of PE material. Both were supplied by the State Grid (China). The insulating material was crosslinked using dicumyl peroxide (DCP) as the crosslinking agent was supplied by Sigma-Aldrich. For bulk material, both LDPE and DCP $(2 \%)$ were processed at temperature of $120^{\circ} \mathrm{C}$ for $15 \mathrm{~min}$ using Thermo PolyLab mixer.

\section{B. Sample Preparation}

The samples used for PEA measurements were of a planer configuration and consisted of XLPE. The electrode arrangements for PEA measurements are shown in Fig. 1; where $A$ represent the conventional setup, with unbonded Sc electrode used as the anode and $\mathrm{Al}$ as the cathode, $B$ is thermally bonded $\mathrm{Sc}$ and $C$ is gold coated. In all cases, the XLPE insulating material remained the same.

Both the insulating sample ( $250 \mu \mathrm{m}$ thickness) and the semicon ( $\sim 50 \mu \mathrm{m}$ thickness) were separately prepared at a 
temperature below the activation threshold of the crosslinking agent. Crosslinking was achieved by hot-pressing the electrodes into the insulating bulk at $200{ }^{\circ} \mathrm{C}$ for $10 \mathrm{~min}$, ensuring a good interfacial bond. The resultant thickness of the samples was approximately $300 \mu \mathrm{m}$. The applied load was maintained during the cooling process and the samples were removed from the hot press once $120^{\circ} \mathrm{C}$ was reached and further cooled down at room temperature. Unbonded sample thickness was $130 \mu \mathrm{m}$.

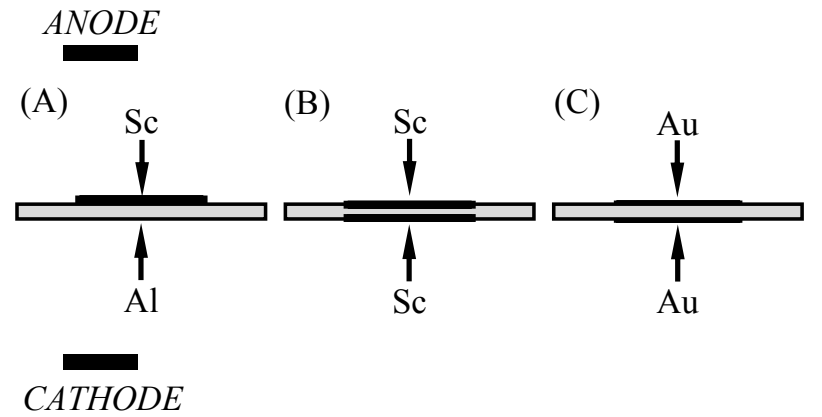

Fig. 1. Schematic illustration of the electrode setups used for PEA measurements. Note, $\mathrm{Sc}$ is the semiconductive material, $\mathrm{Al}$ is aluminum and $\mathrm{Au}$ is gold; the bulk XLPE insulating material remained the same in all cases.

\section{Degassing}

It is well known that crosslinking agents produces byproducts within the material as a result of the crosslinking reactions [8,9]. Therefore, all samples were degassed in a vacuum oven for $6 \mathrm{~d}$ at a constant temperature of $70{ }^{\circ} \mathrm{C}$ to remove volatile residual byproducts from the crosslinked samples.

\section{Space Charge Measurement}

The space charge evolution with the different electrode systems was measured using the PEA technique. For this, the sample was inserted between the lower and upper electrodes and an electric field of $40 \mathrm{kV} / \mathrm{mm}$ was applied. Space charge data were acquired both with the field applied to the sample and during its subsequent decay under short circuit condition. The evolution of charge, polarization (voltage on) and depolarization (voltage off) for each stressing period lasted 60 min. All PEA tests were carried out at room temperature.

It is well known that the PEA technique is an indirect method of space charge profile measurement and, therefore, to obtain quantitative charge distributions, calibration is essential $[2,10]$. Therefore, calibration was done for all PEA tests by applying a low voltage $(2 \mathrm{kV})$ for a short period of time $(5$ seconds) to show the capacitive charges on both electrodes. This low voltage was chosen to ensure no space charge is develops in the bulk sample.

\section{RESULTS}

Fig. 2 shows result obtained from a sample with $\mathrm{Al}$ as the cathode and unbonded Sc as the anode; heterocharge is observed adjacent to the Al electrode and no charge in the bulk sample. From those later, it would seem that the Sc electrode enhances positive charge injection at the anode.

Fig. 3 shows space charge profiles obtained from the sample with bonded Sc as the anode and cathode. It can be seen that positive charge injection is further enhanced in comparison with the unbonded Sc case shown in Fig. 2. However, little difference is observed at the cathode, except that, as the magnitude is slightly higher for the thermally bonded Sc (Fig. 3). Moreover, some charges are evident in the bulk.

If $\mathrm{Au}$ replaces both electrodes, shown in Fig. 4, the formation of heterocharge can be observed in both the cathode and the anode and the accumulation of heterocharge is clearly reflected in the volts off space charge profiles.

For the conventional PEA setup shown in Fig. 2, the charge amount is somewhat lower than those observed in the samples using either bonded $(\mathrm{Sc})$ semicon or coated ( $\mathrm{Au}$ ) electrodes. In all cases, significant amounts of charge adjacent to the cathode are observed in both the voltage-on and the decay process. It can be seen in Fig.2 that the magnitude of heterocharge (positive peak) adjacent to the cathode is $\sim 4.5 \mathrm{Cm}^{-3}$ for the volts-on test and slightly higher $\left(5.3 \mathrm{Cm}^{-3}\right)$ once the external voltage was removed at $30 \mathrm{~s}$ (Fig. 2b). This phenomenon is also observed in the other two cases; such as, bonded Sc (Fig. 3a) and gold coated electrode (Fig. 4a), the heterocharge peaks at $60 \mathrm{~min}$ is $5.7 \mathrm{Cm}^{-3}$ and $7.6 \mathrm{Cm}^{-3}$, respectively. However, the magnitude of the peaks shortly after the removal of voltage is increased to $9.5 \mathrm{Cm}^{-3}$ (Fig. 3b) and $10.3 \mathrm{Cm}^{-3}$ (Fig. 4b). These effects are further explained in the discussion section.
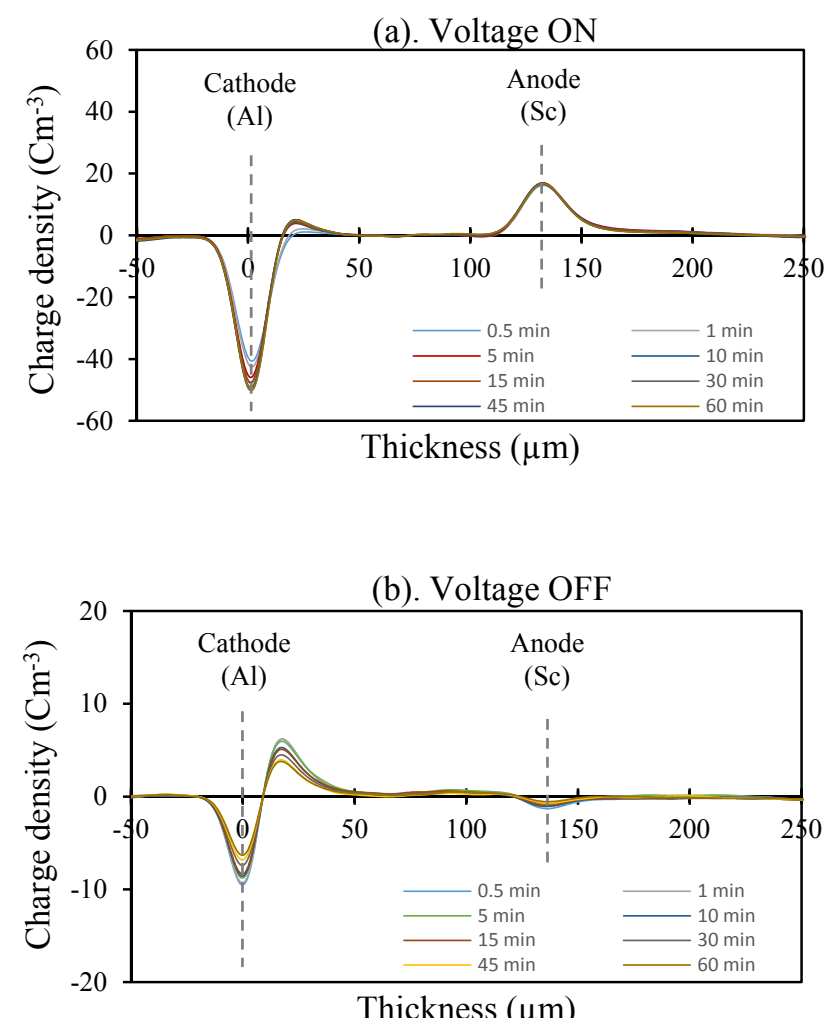

Fig. 2. PEA charge density profile of XLPE using unbonded Sc/Al electrodes. The applied voltage was $40 \mathrm{kV} / \mathrm{mm}$ at room temperature. 


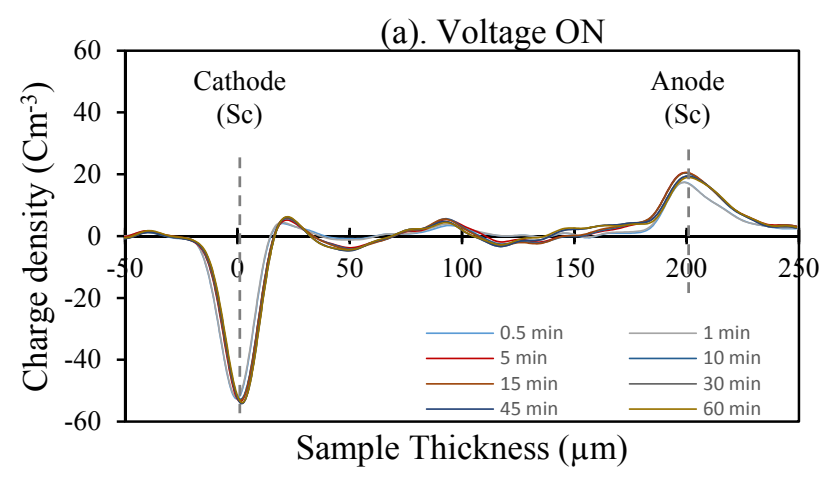

(b). Voltage OFF

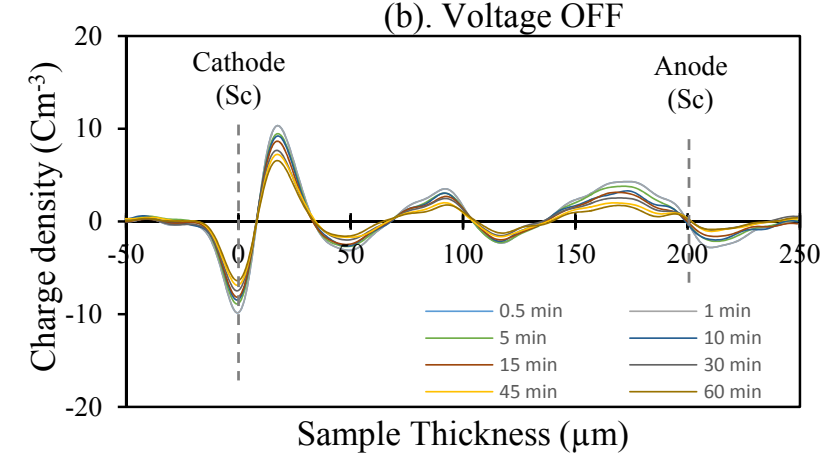

Fig. 3. PEA charge density profile of XLPE thermally bonded Sc electrodes. The applied voltage was $40 \mathrm{kV} / \mathrm{mm}$ at room temperature.

(a). Voltage ON

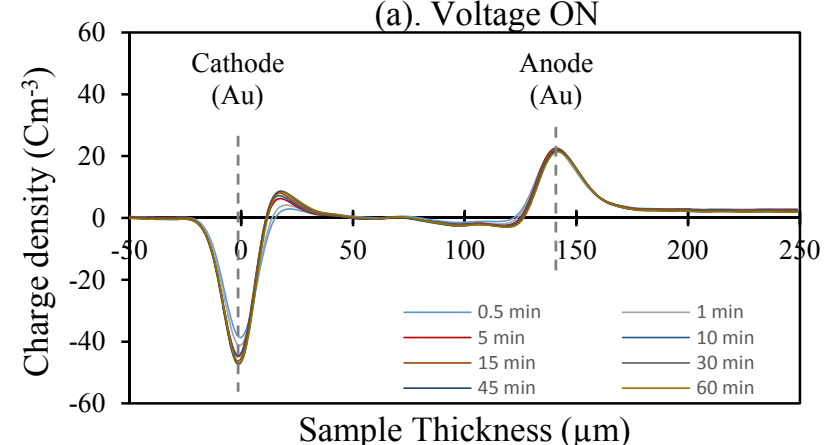

(b). Voltage OFF

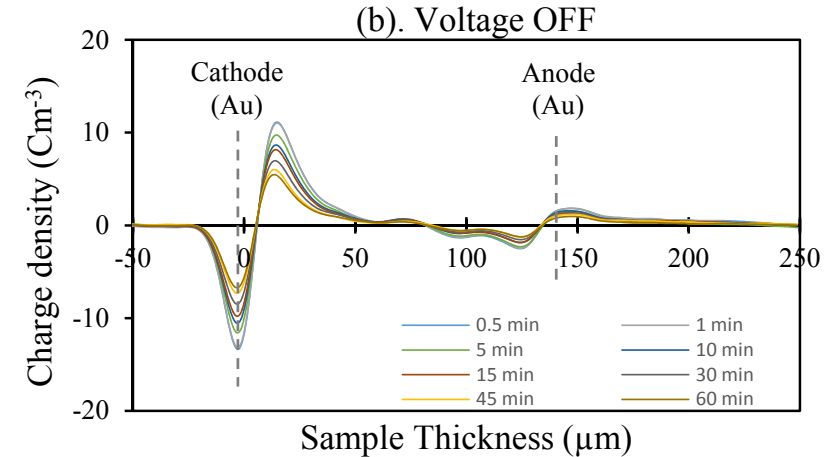

Fig. 4. PEA charge density profile of XLPE/Au electrodes. The applied voltage was $40 \mathrm{kV} / \mathrm{mm}$ at room temperature.

\section{DISCUSSION}

In the present work, different combination of electrodes was used for PEA measurements, and the individual effect of these electrode materials on charge injection was examined. The PEA results clearly demonstrate that the electrode materials have a significant effect on charge injection. By examining the space charge distributions in XLPE bulk with different electrode combinations either with volts on or off, the results clearly show the electrode effect on charge injection. In all cases, similar charge injection was observed close to the cathode; however, the amount of charge in the bulk (Fig. 2) is smaller when compared with the bonded Sc and $\mathrm{Au}$ electrodes. Referring to the results section, the following effects on space charge profile were observed.

(i) When $\mathrm{Al}$ acts as the cathode and $\mathrm{Sc}$ as the anode, the Sc enhances positive charge injection in system, mostly at the anode (Fig. 2).

(ii) When bonded Sc acts as the cathode and the anode, a further enhancement in positive charge injection and some charges trapped in the bulk were observed. However, not much difference was observed at the cathode (Fig. 3).

(iii) When $\mathrm{Au}$ act as the cathode and the anode, accumulation of heterocharge occurred at both electrodes (Fig. 4).

With bonded Sc electrodes (Fig. 3), it can be seen that positive charge is formed across the sample with the maximum adjacent to the cathode. The amount and magnitude of both negative and positive charge are significantly higher than those observed for the $\mathrm{Al}$ electrode. These findings are in agreement with those previously observed in LDPE [11]. In Fig. 3, heterocharge built up adjacent to the cathode and homocharge started to form adjacent to the anode, suggesting dominant positive charge injection at the anode. Since a large amount of charge can be injected from both Sc electrodes, this leads to a significant amount of charge build up in the bulk material.

Although, the bulk XLPE samples were degassed to remove volatile byproducts, there is a possibility that some of these byproducts may have remained within the material. In such a case, two possible scenarios can be imagined. First, the space charge formation within the insulation material is caused by the ionization of residual crosslinking byproducts leftover from the crosslinking process and the injection from electrodes are trapped across the insulation material. Similar observation were made by Choo et al [3] in their studies on space charge accumulation under DC stress for XLPE insulating material and it was found that the heterocharge formation occurred due to ionic carriers supplied from impurities. In another study it was also observed that homocharge and heterocharge formation occurred in XLPE [12], suggesting that the crosslinking byproducts (impurities) may become charged and migrate to the counter electrode under DC stress [3]. Therefore, it can be suggested that the crosslinking byproducts will undergo an ionization process under high electric field and these ionized species will move towards the opposite electrode to form heterocharge. 
Alternatively, with thermally bonded crosslinked semicon and XLPE materials, it is possible that diffusion of carbon black particle may have occurred from the Sc into the polymer during the sample preparation. As a result, it has changed the chemical composition of the insulating material, thereby facilitating heterocharge and homocharge accumulation at both electrodes. Gold coated electrode in Fig. 4 exhibits heterocharge formation at both electrodes; this means the diffusion may not be the case in XLPE sample; as with the bonded semicon positive charge injection dominated and resulted in homocharge formation at the anode.

Referring to the results, both charge injection and ionization occur in the bulk at the same time during the voltage application. These charges may have overlapped at the cathode region. As only net charges can be measured by the PEA system, therefore, the increase of positive charges adjacent to the cathode on the removal of voltage can be observed due to the fast decay of injected negative charges, such as, only positive charges remain in the system. This suggests that more negative charges are injected from the cathode, thereby, greater variation is observed between the voltage-on and voltage-off results. To further clarify this effect, the amount of injected negative charges from the cathode in a conventional case are $0.8 \mathrm{Cm}^{-3}$ (Fig. 2), gold coated $2.7 \mathrm{Cm}^{-3}$ (Fig. 3) and bonded Sc $3.8 \mathrm{Cm}^{-3}$ (Fig. 4). This indicates the gold coated and bonded electrodes enhance the charge injection and the injection rate from the semicon is higher than that of metallic electrode. In Ref [11], it has been shown that the Sc electrode injects charge more easily than Al electrode. As stated earlier, the semicon used in the current study was HVDC cable grade carbon loaded XLPE; this should have similar properties to the bulk XLPE insulating material. Therefore, the injection would have been much easier than that of metallic electrodes. Since the charge injection rate of $\mathrm{Al}$ and $\mathrm{Au}$ electrode is lower than that of Sc [13], therefore, the amount of charge in the bulk samples would be less, reflects finding of Fig. 2 and Fig.4.

\section{CONCLUSIONS}

The effect of electrode material on space charge accumulation in XLPE has been investigated. The results demonstrate that the electrodes play a significant role in charge injection and that thermally bonded semicon enhances charge injection at both electrodes. The experimental result demonstrate that the nature of the semiconducting material has an important role in space charge accumulation in the XLPE bulk, affecting injection and extraction of charges. The underlying mechanisms such as diffusion of materials are not clear yet and require further investigation.

Space charge studies in polymeric insulation materials for both scientific and technological advancement is an important area to be understood for the development of future HVDC cable technologies. These findings can be exploited for HVDC XLPE polymeric cable industries. In fact, the space charge measurements conducted in this study could provide fundamental information for selecting the right electrode/insulation combination.

\section{ACKNOWLEDGMENT}

The authors are grateful for the financial support from the State Grid Cooperation of China: Research on Key Technologies of Insulation Material and Accessories for $320 \mathrm{kV} \mathrm{HVDC} \mathrm{XLPE}$ Cable System (SGRIZLJS(2014)888).

\section{REFERENCES}

[1] T. Yamanaka, S. Maruyama, and T. Tanaka, "The development of DC+/$500 \mathrm{kV}$ XLPE cable in consideration of the space charge accumulation," in Properties and Applications of Dielectric Materials, 2003. Proceedings of the 7th International Conference on, 2003, pp. 689-694 vol.2.

[2] M. Fu, L. A. Dissado, G. Chen, and J. C. Fothergill, "Space charge formation and its modified electric field under applied voltage reversal and temperature gradient in XLPE cable," IEEE Transactions on Dielectrics and Electrical Insulation, vol. 15, pp. 851-860, 2008.

[3] W. Choo, G. Chen, and S. G. Swingler, "Electric field in polymeric cable due to space charge accumulation under DC and temperature gradient," IEEE Transactions on Dielectrics and Electrical Insulation, vol. 18, pp. 596-606, 2011.

[4] Z. Yewen, J. Lewiner, C. Alquie, and N. Hampton, "Evidence of strong correlation between space-charge buildup and breakdown in cable insulation," IEEE Transactions on Dielectrics and Electrical Insulation, vol. 3, pp. 778-783, 1996.

[5] N. Hozumi, H. Suzuki, T. Okamoto, K. Watanabe, and A. Watanabe, "Direct observation of time-dependent space charge profiles in XLPE cable under high electric fields," IEEE Transactions on Dielectrics and Electrical Insulation, vol. 1, pp. 1068-1076, 1994.

[6] R. Bartnikas, "Performance characteristics of dielectrics in the presence of space charge," IEEE Transactions on Dielectrics and Electrical Insulation, vol. 4, pp. 544-557, 1997.

[7] G. Blaise, "Space-charge physics and the breakdown process," Journal of Applied Physics, vol. 77, pp. 2916-2927, 1995.

[8] Y. L. Chong, G. Chen, H. Miyake, K. Matsui, Y. Tanaka, and T. Takada, "Space charge and charge trapping characteristics of cross-linked polyethylene subjected to ac electric stresses," Journal of Physics D: Applied Physics, vol. 39, pp. 1658-1666, 2006.

[9] N. Hussin and G. Chen, "The trapping characteristic of low density polyethylene in the presence of crosslinking by-products," Journal of Physics: Conference Series, vol. 183, p. 012007, 2009.

[10] G. Chen, Y. L. Chong, and M. Fu, "Calibration of the pulsed electroacoustic technique in the presence of trapped charge," Measurement Science \& Technology, vol. 17, pp. 1974-1980, 2006.

[11] G. Chen, T. Y. G. Tay, A. E. Davies, Y. Tanaka, and T. Takada, "Electrodes and charge injection in low-density polyethylene using the pulsed electroacoustic technique," IEEE Transactions on Dielectrics and Electrical Insulation, vol. 8, pp. 867-873, 2001.

[12] F. Davide, M. Gian-Carlo, D. Leonard.A, L. Christian, T. Gilbert, M. Peter.H. F, B. Riccardo, C. Alfred, and N. Ulf. H, "Effect of semiconinsulation interface on space charge formation in HVDC polymeric cables," in 7th International Conference on Power Insulated Cables (JICABLE-07), Paris, 2007.

[13] G. Chen, Y. Tanaka, T. Takada, and L. Zhong, "Effect of polyethylene interface on space charge formation," IEEE Transactions on Dielectrics and Electrical Insulation, vol. 11, pp. 113-121, 2004. 